\title{
Dynamical and Structural Properties of Water in Silica Nanoconfinement - Impact of Pore Size, Ion Nature and Electrolyte Concentration
}

Markus Baumt, Francois Rieutord", Fanni Juranyi*, Cyrielle Reyt, Diane Rébiscoul†

† CEA, ICSM - UMR 5257 CEA-CNRS-UM-ENSCM, 30207 Bagnols-sur-Cèze Cedex,

France

${ }^{*}$ Paul-Scherrer-Institute, 5232 Villigen PSI, Switzerland

₹ Univ. Grenoble Alpes, INAC-MEM, F-38000 Grenoble, France

〜Corresponding author: diane.rebiscoul@cea.fr

\section{Supplementary information}

The final $\mathrm{pH}$ values of the elecrtolyte solutions $\mathrm{XCl}_{2}$ (with $\mathrm{X}=\mathrm{Ba}^{2+}, \mathrm{Ca}^{2+}$ and $\mathrm{Mg}^{2+}$ ) measured at the laboratory atmosphere.

Table SI1: $\mathrm{pH}$ values of electrolyte solutions $\mathrm{XCl}_{2}$ (with $\mathrm{X}=\mathrm{Ba}^{2+}, \mathrm{Ca}^{2+}$ and $\mathrm{Mg}^{2+}$ ) measured at laboratory atmosphere. The uncertainties do not exceed \pm 0.05 .
$\left[\mathrm{XCl}_{2}\right]_{0}$
$0.01 \mathrm{M}$
$0.2 \mathrm{M}$
$1 \mathrm{M}$ 


\begin{tabular}{llll}
\hline \hline $\mathrm{BaCl}_{2}$ & 5.79 & 5.47 & 5.38 \\
$\mathrm{CaCl}_{2}$ & 6.22 & 6.31 & 6.37 \\
$\mathrm{MgCl}_{2}$ & 6.35 & 6.07 & 5.77 \\
\hline
\end{tabular}

\section{MCM-41 and grafted MCM-41}
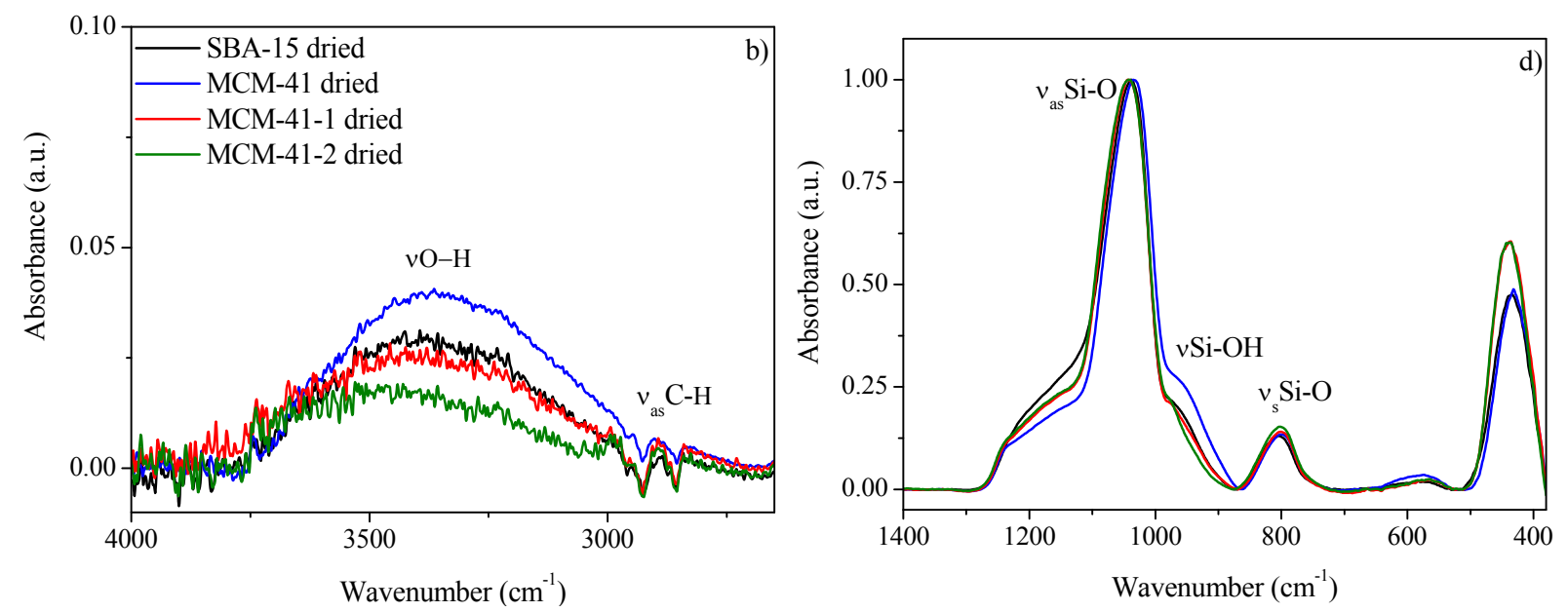

Figure SI1: FTIR-ATR spectra showing the characteristic $v \mathrm{OH}, v_{\mathrm{as}} \mathrm{CH}$ bands and $v \mathrm{SiO}$ bands of dried SBA-15, MCM-41 and grafted MCM-41. The analysis were made at the laboratory atmosphe, i.e. without humidity control. The presence of vasC-H bands is related to the contamination of the ATR crystal, as already observed in previous studies ${ }^{27,28}$.

Figure SI2 shows the SAXS pattern of MCM-41 and grafted MCM-41. The pattern of MCM-41 presents a strong and well-defined peak at $\mathrm{Q}=1.55 \mathrm{~nm}^{-1}$ and three peaks of weaker intensity at around $2.67 \mathrm{~nm}^{-1}$ and $3.09 \mathrm{~nm}^{-1}$ and at $\mathrm{Q}=4.01 \mathrm{~nm}^{-1}$. These peaks correspond to the (100), (110), (200) and (300) lattice planes of the hexagonal MCM-41 structure. For MCM-41-1 (MCM-41-2) the peaks are slightly shifted: $\mathrm{Q}_{1}=1.62 \mathrm{~nm}^{-1}\left(1.72 \mathrm{~nm}^{-1}\right), \mathrm{Q}_{2}=2.78 \mathrm{~nm}^{-1}$ 
$\left(2.90 \mathrm{~nm}^{-1}\right), \mathrm{Q}_{3}=3.23 \mathrm{~nm}^{-1}\left(3.35 \mathrm{~nm}^{-1}\right)$, and $\mathrm{Q}_{4}=4.20 \mathrm{~nm}^{-1}\left(4.26 \mathrm{~nm}^{-1}\right)$. From these data, interreticular distances $d$ were calculated by using eq. 1 :

$$
d=\frac{\lambda}{2 \sin \theta}
$$

where $\lambda$ is the wavelength of incident wave; $d$ is the spacing between the planes in the mesoporous lattice; and $\theta$ is the angle between the incident beam and the scattering planes. The unit cell parameter $a_{0}$ was also deduced from eq. 1 and 2:

$$
a_{0}=\frac{2 d_{100}}{\sqrt{3}}
$$
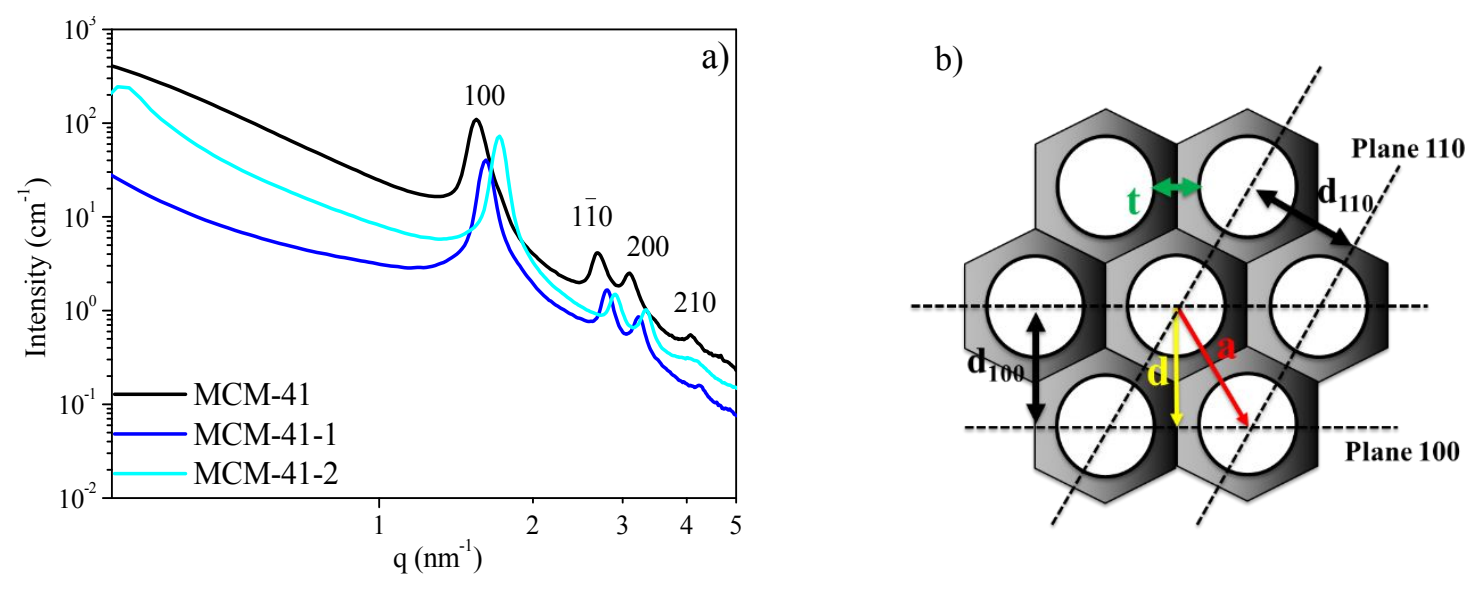

Figure SI2 : Small-Angle X-Ray Scattering spectra of MCM-41 and grafted MCM-41 show the Bragg peaks. The right panel presents the various parameters defining the hexagonal arrangement of the mesopores. 

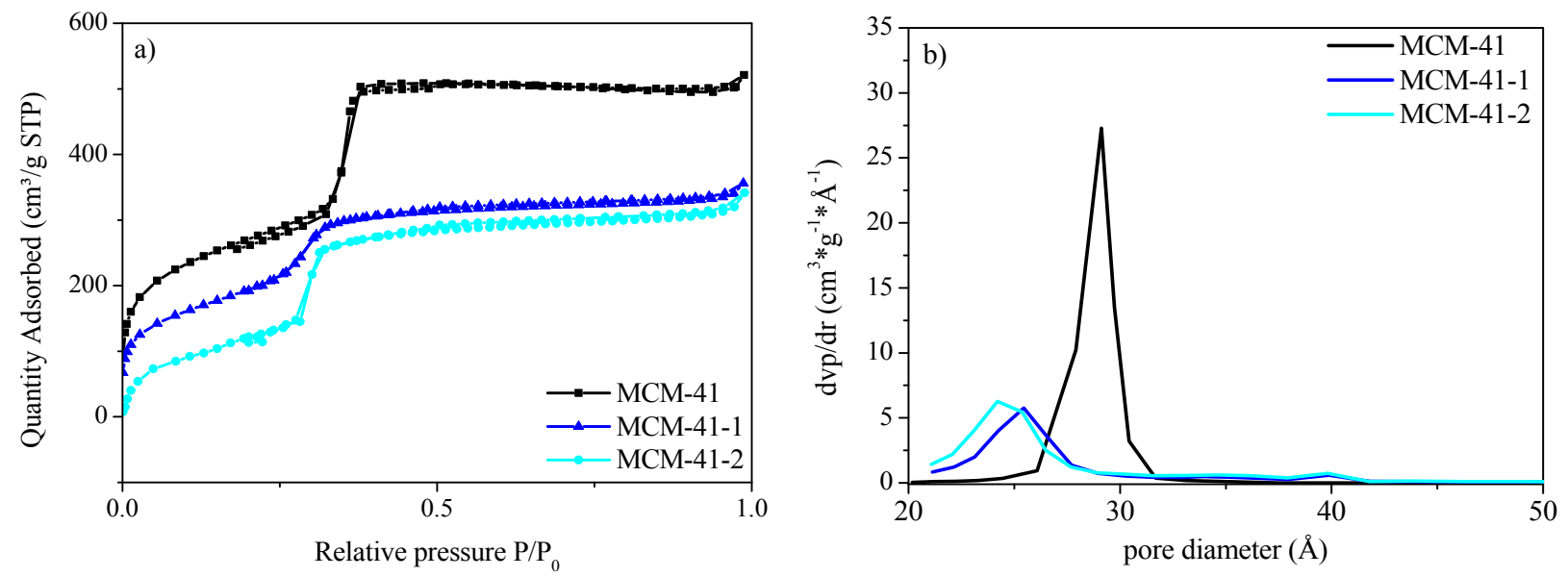

Figure SI3: Nitrogen adsorption-desorption isotherms (a) and pore size distributions of MCM-41 and grafted MCM-41 (b). 


\section{Sorption isotherms}
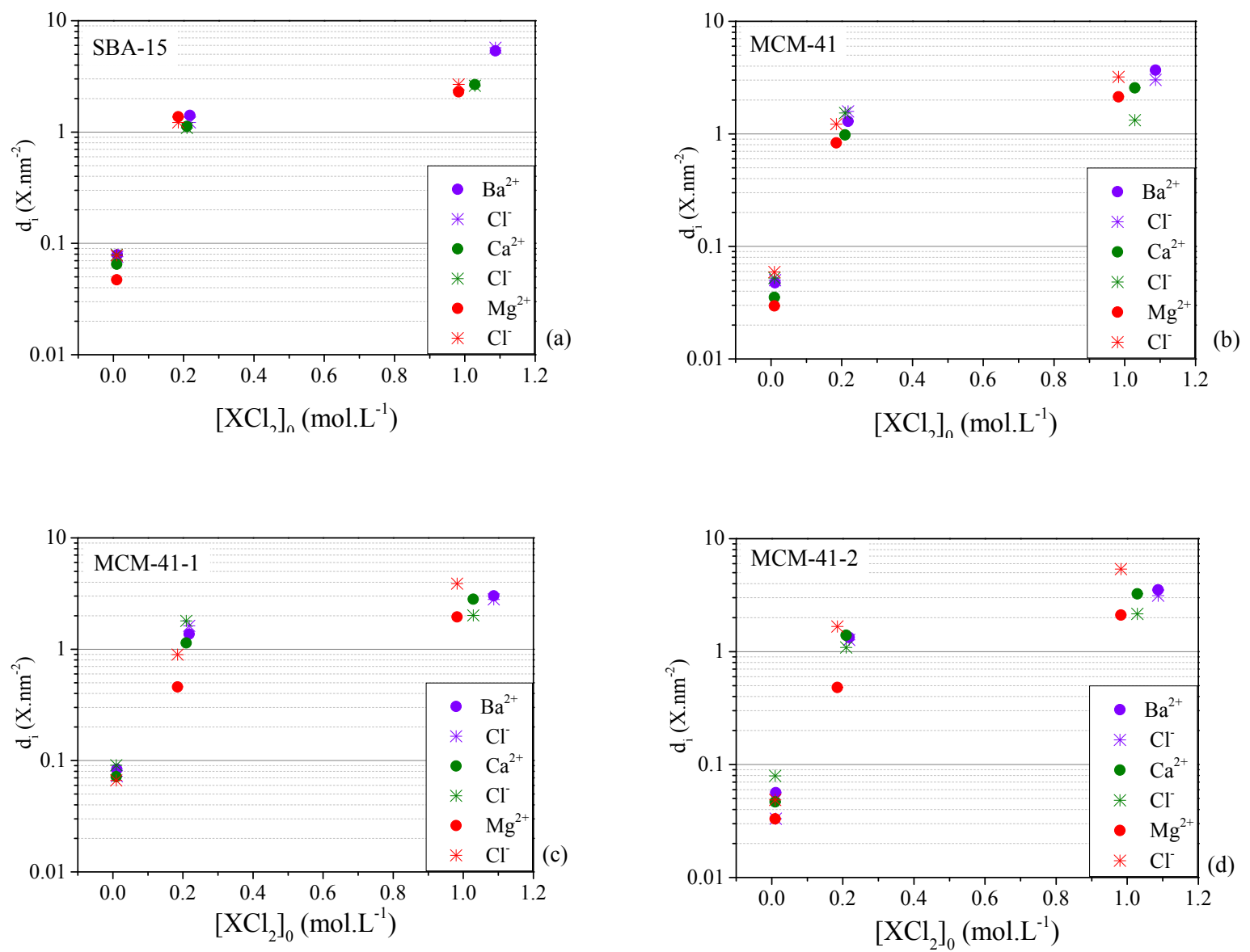

Figure SI4: Surface ion excess $d_{i}$ in SBA-15, MCM-41 and grafted MCM-41 as a function of the initial electrolyte concentration. 


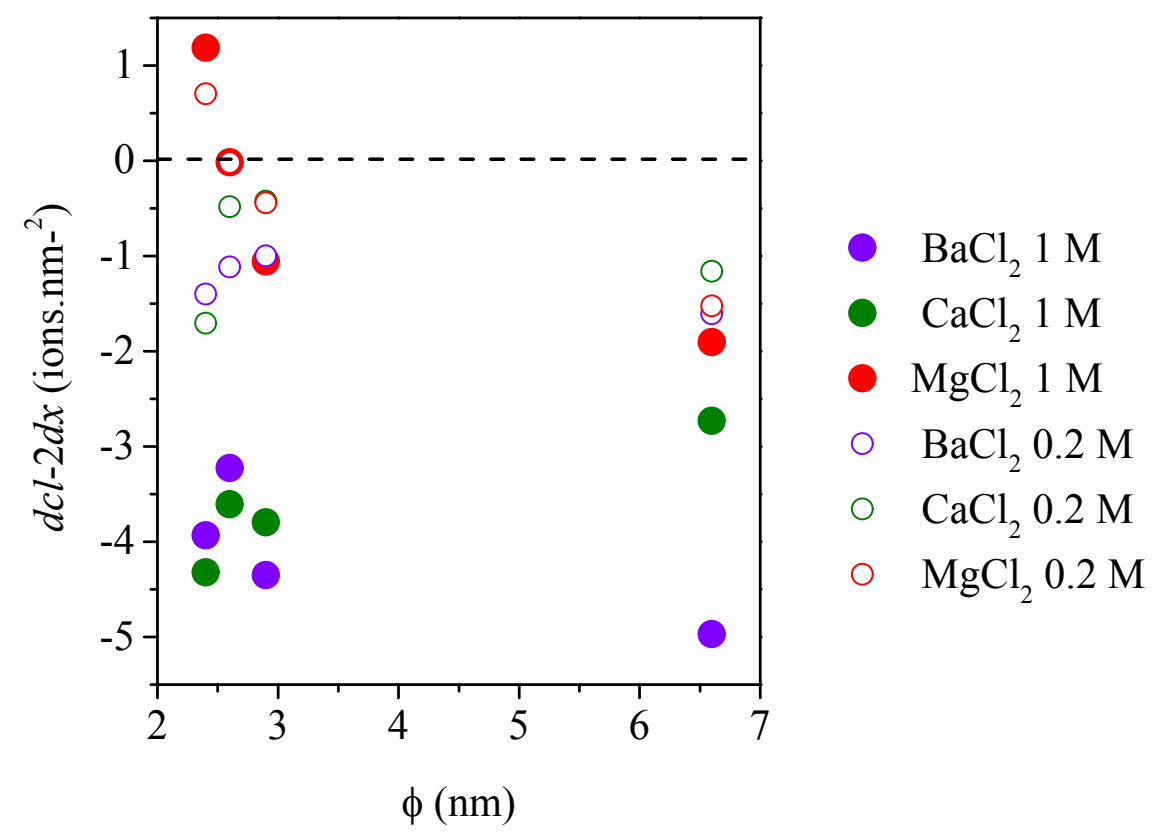

Figure SI5: Evolutions of $d_{C l}-2 d_{X}$ at the equilibrium of the sorption isotherms for various electrolyte solutions as a function of the mean pore size $\phi_{p}$. 


\section{Decomposition of O-H stretching band in Infrared Spectroscopy}

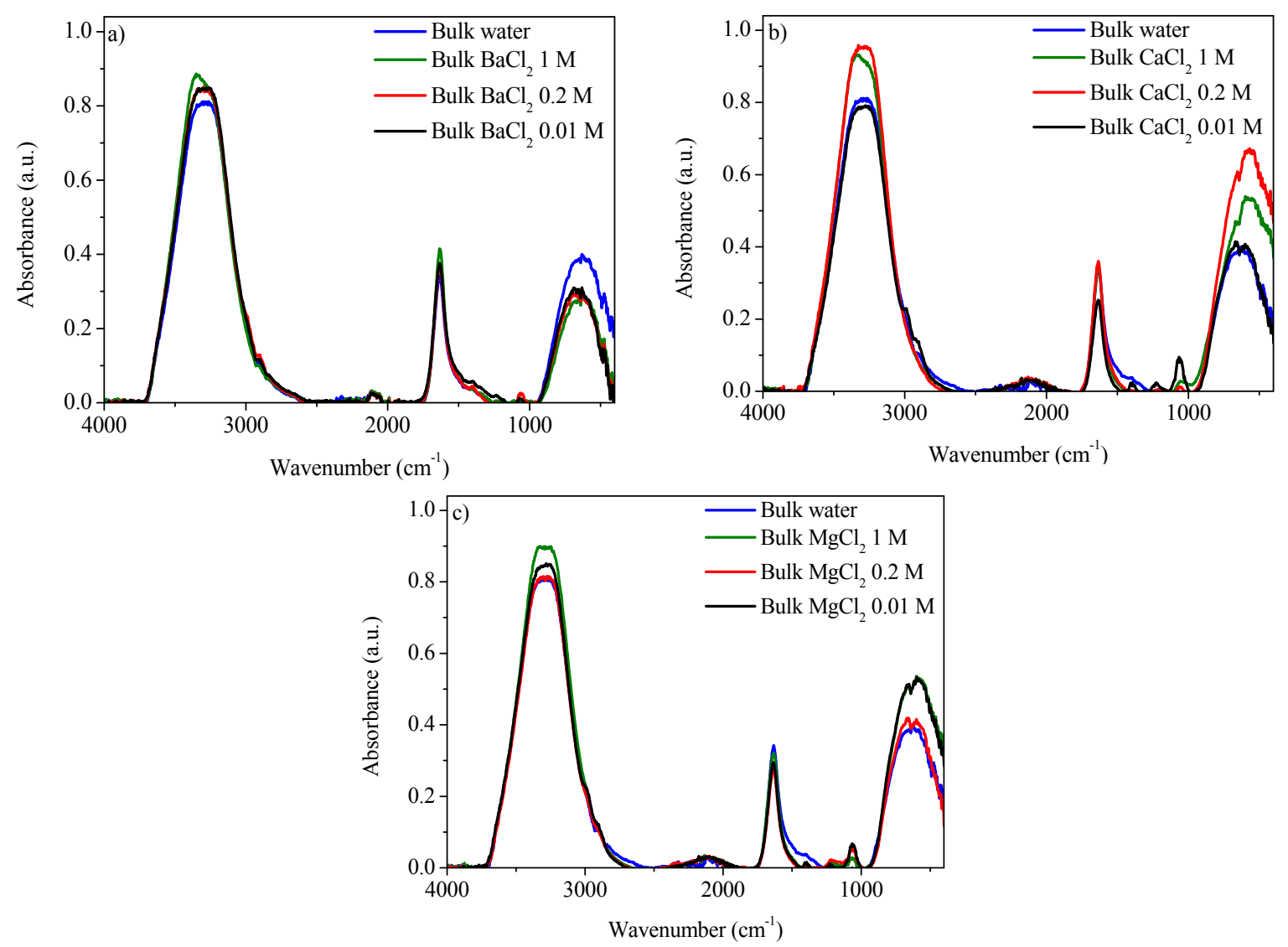

Figure SI6: Experimental ATR-FTIR spectra of bulk solutions (a) to (c). 

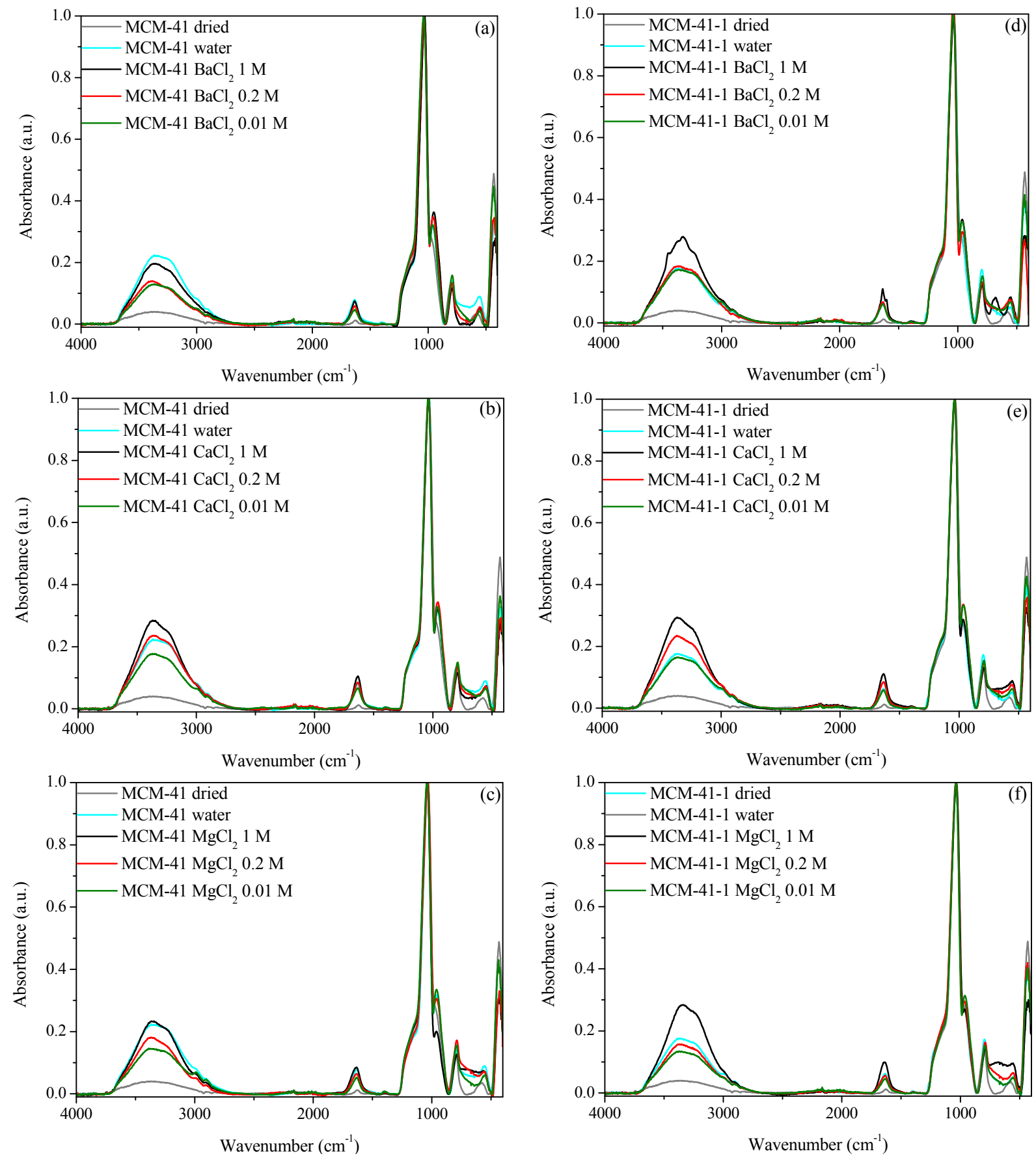

Figure SI7: Experimental ATR-FTIR spectra of MCM-41 (a) to (c) and MCM-41-1 (d) to (f) filled with water and electrolyte solution at various concentrations. 
Table SI2: Percentages of water in the hydration shell of ions present in electrolyte solutions.

\begin{tabular}{ccccc}
\hline Electrolytes & $\begin{array}{c}\text { Hydration } \\
\text { number }\end{array}$ & $\begin{array}{c}0.01 \mathrm{M} \\
(\%)\end{array}$ & $\begin{array}{c}0.2 \mathrm{M} \\
(\%)\end{array}$ & $\begin{array}{c}1 \mathrm{M} \\
(\%)\end{array}$ \\
\hline \hline $\mathrm{Mg}^{2+}$ & 6.0 & 0.01 & 2.2 & 11.3 \\
\hline $\mathrm{Ca}^{2+}$ & 7.0 & 0.01 & 2.5 & 13.1 \\
\hline $\mathrm{Ba}^{2+}$ & 9.5 & 0.02 & 3.4 & 17.2 \\
\hline $\mathrm{Cl}^{-}$ & 5.6 & 0.01 & 2.0 & 10.2 \\
\hline
\end{tabular}
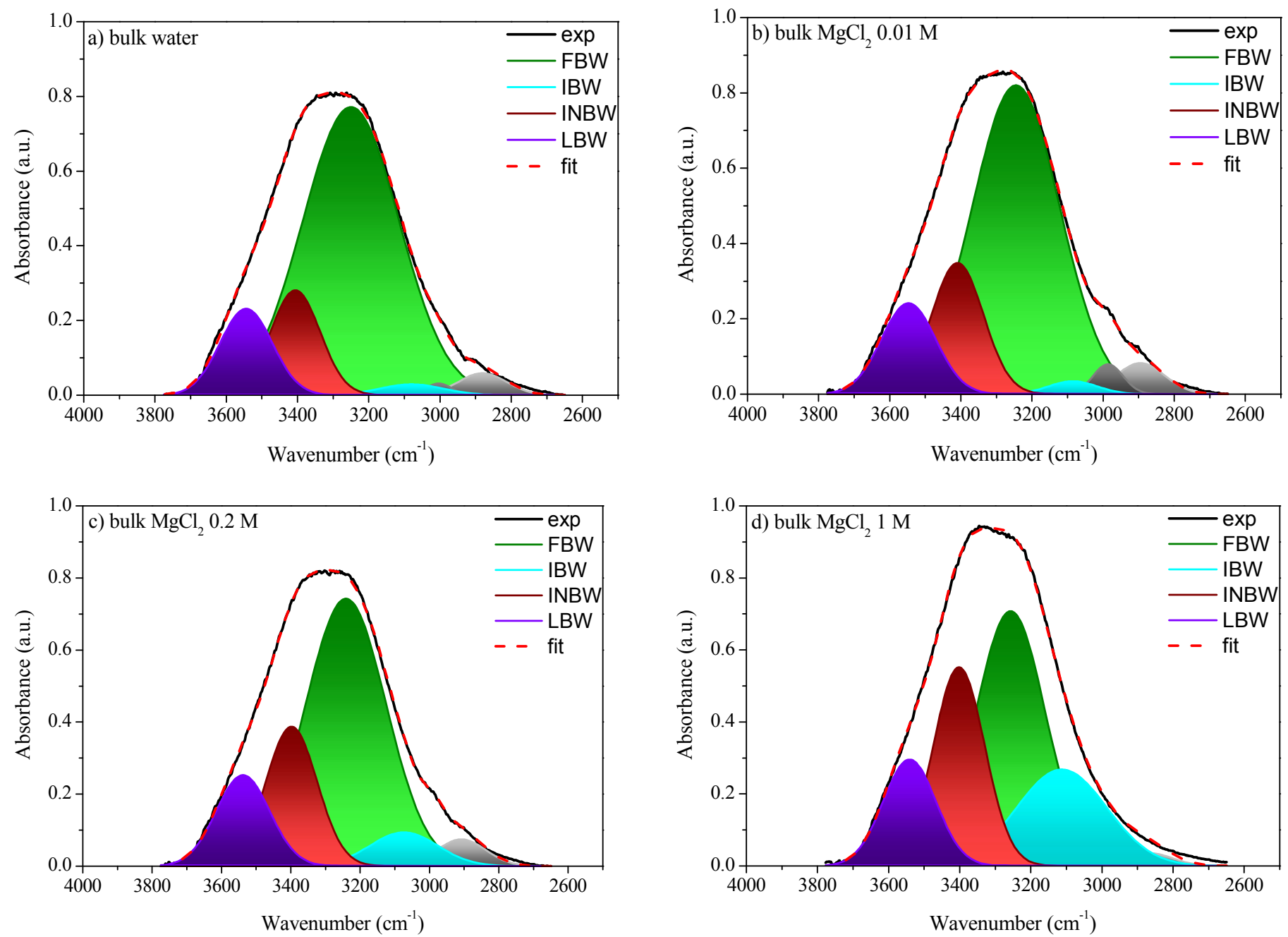

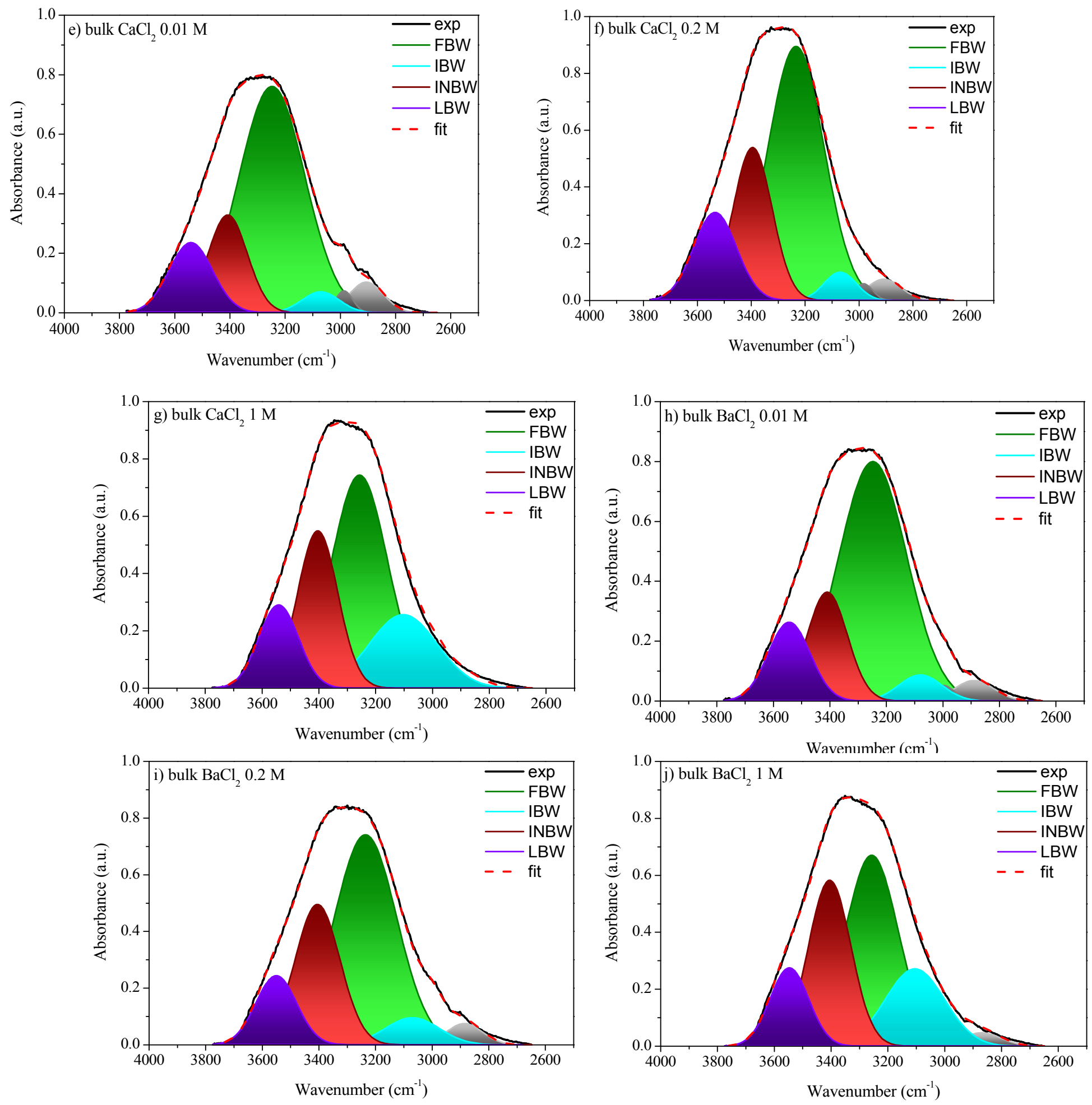

Figure SI8: Decomposition of the $\mathrm{vOH}$ band between 2650 and $3775 \mathrm{~cm}^{-1}$ of bulk electrolyte solutions (a) to (j). 

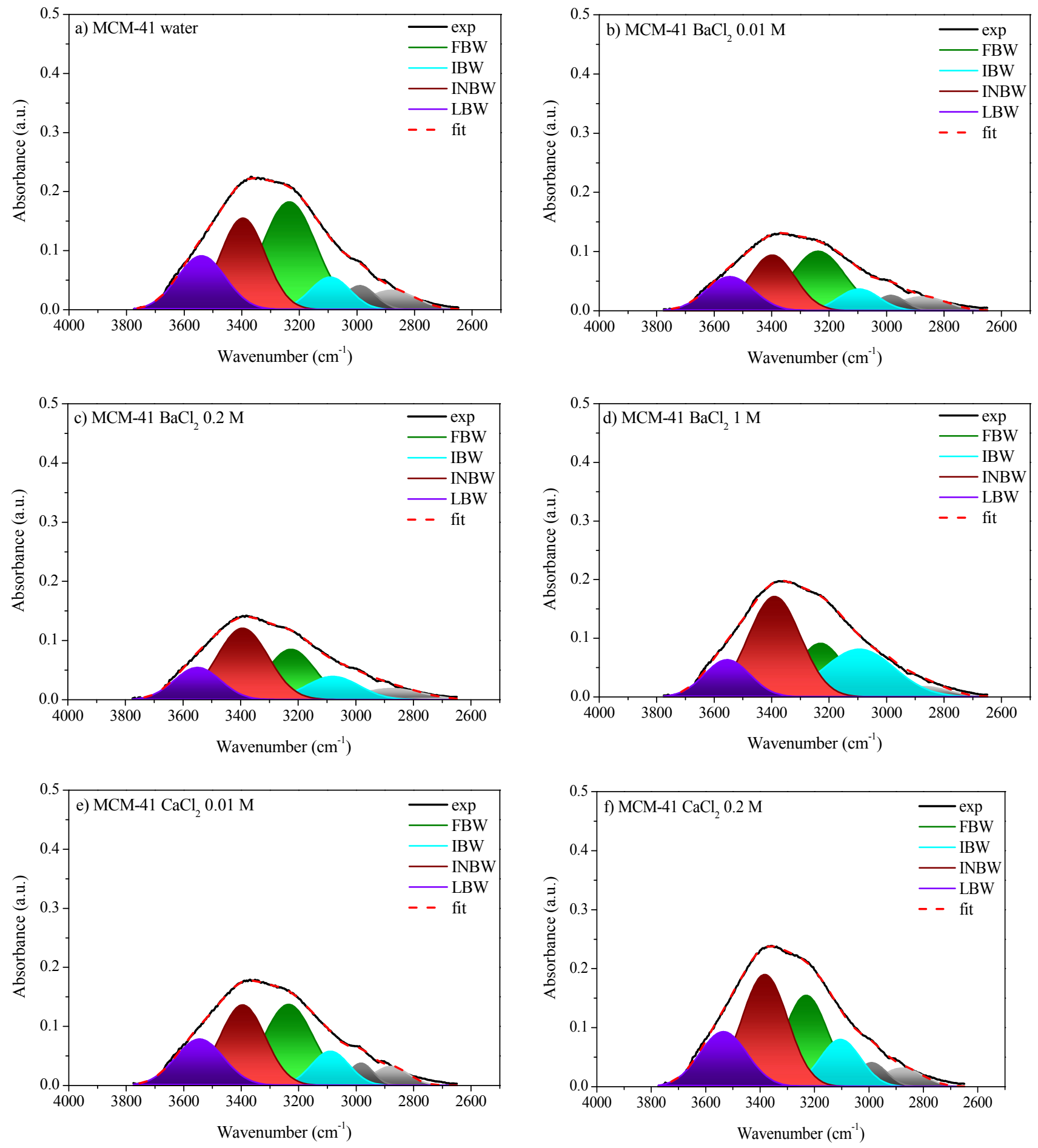

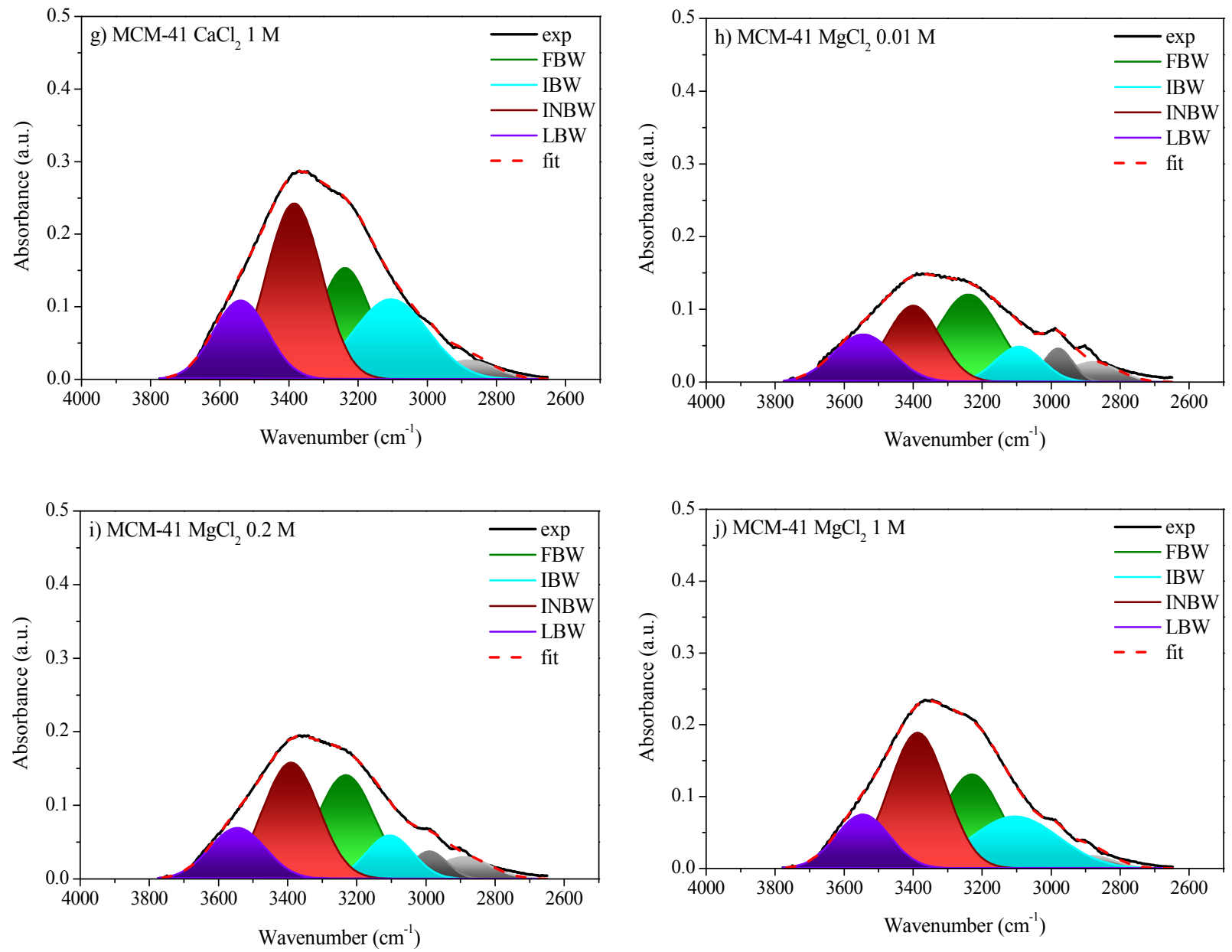

Figure SI9: Decomposition of the $v \mathrm{OH}$ band between 2650 and $3775 \mathrm{~cm}^{-1}$ of electrolyte solutions confined in MCM-41 (a) - (j). 

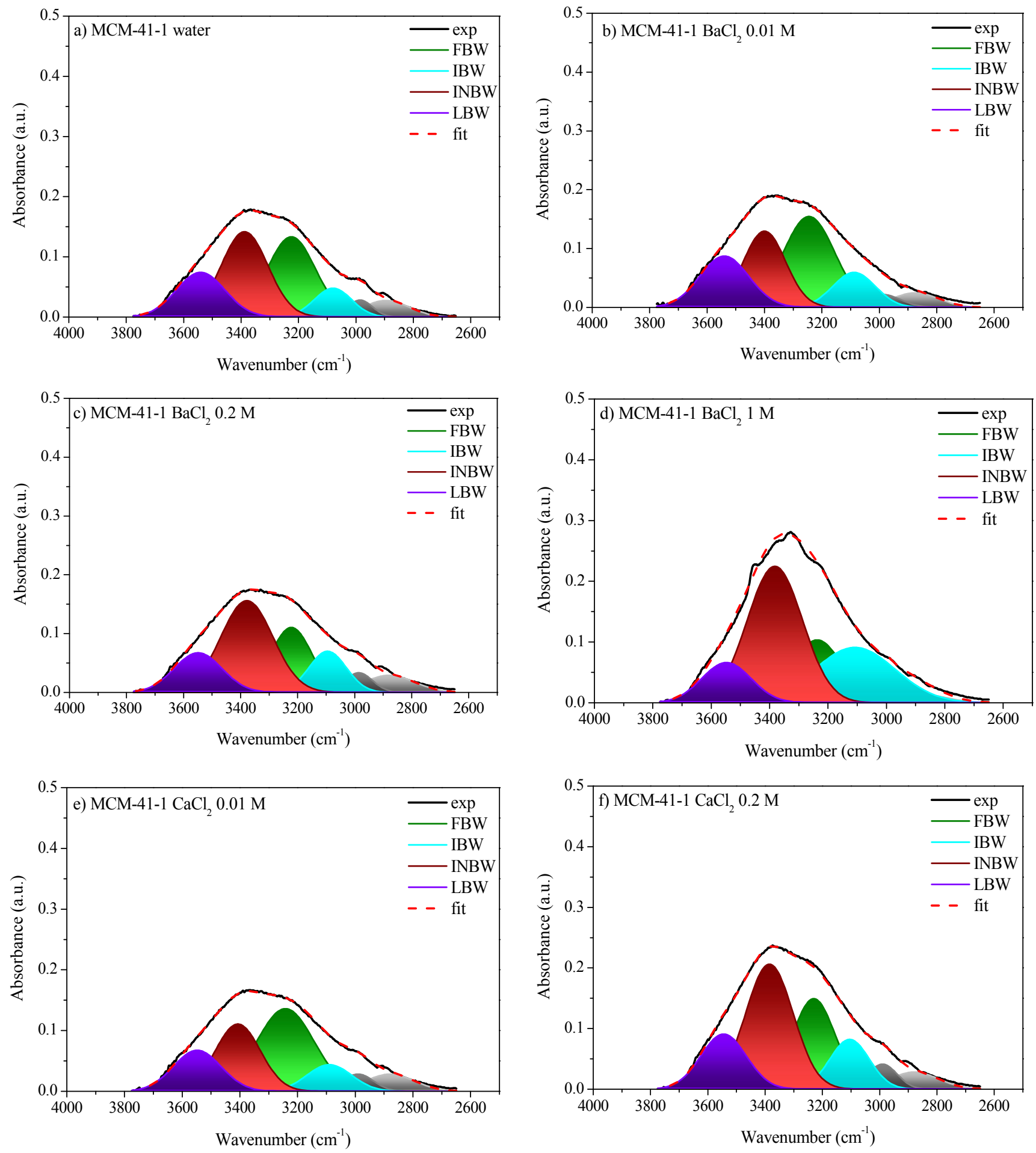

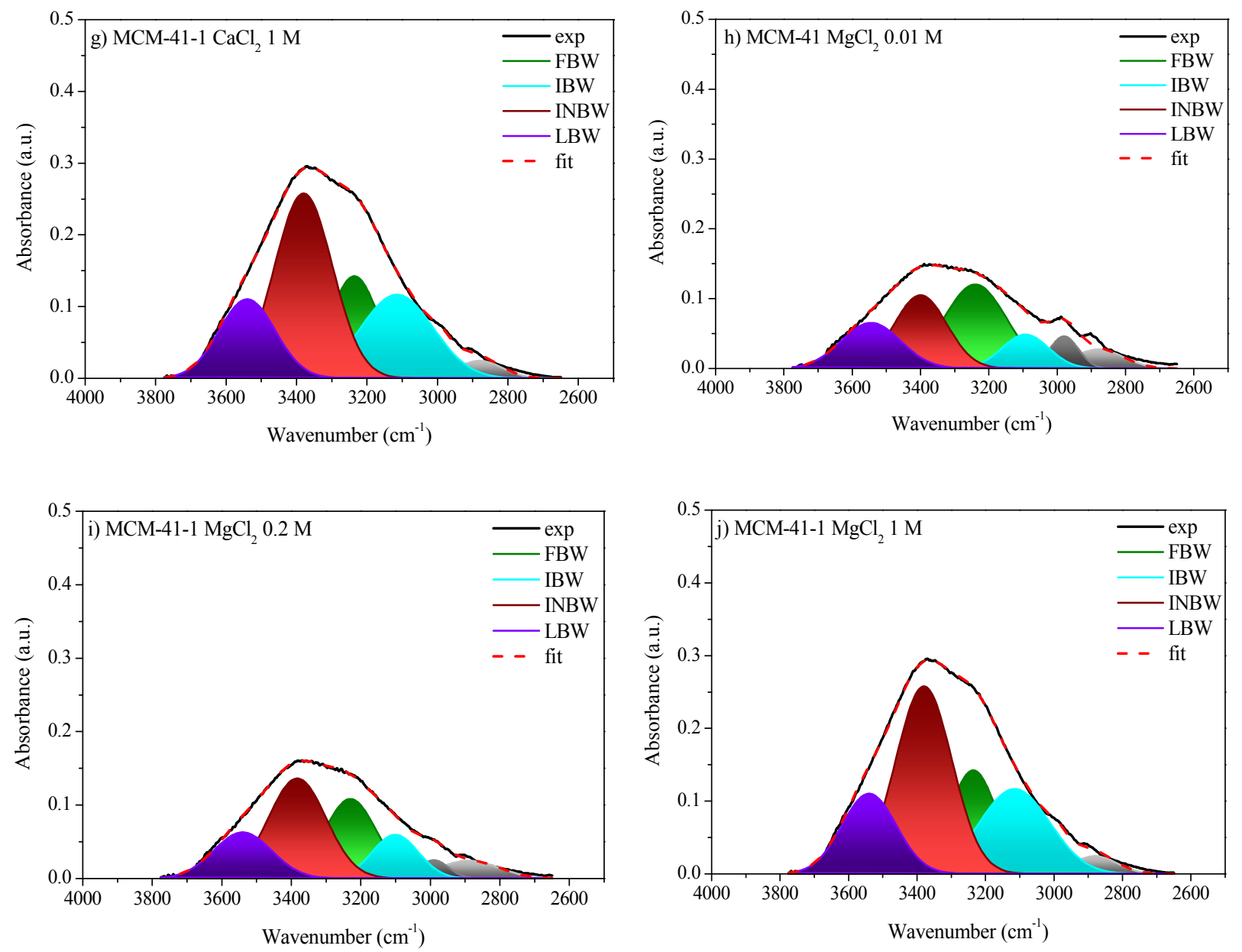

Figure SI10: Decomposition of the $v O H$ band between 2650 and $3775 \mathrm{~cm}^{-1}$ of electrolyte solutions confined in MCM-41-1 (a) - (j).

In some cases, we added two peaks at 2900 and $2990 \mathrm{~cm}^{-1}$ to include the $\mathrm{C}-\mathrm{H}$ vibrations coming from contamination of the spectrometer diamond. The contributions of these two peaks do not exceed $5 \%$ and $4 \%$ of the relative band area, respectively.

The best-fit parameters (center frequencies $\omega$, intensity I and half width at half maxima $\Gamma$ ) of $\mathrm{O}-\mathrm{H}$ stretching band for bulk water, bulk electrolyte solutions and solutions confined in SBA15 are summarized in Table SI2. 
Table SI3: Best-fit parameters (center frequencies $\omega$, intensity I and half width at half maxima $\Gamma$ ) of $\mathrm{O}-\mathrm{H}$ stretching band for bulk water, bulk electrolyte solutions and solutions confined in MCM-41 and grafted MCM-41.

\begin{tabular}{|c|c|c|c|c|c|c|c|c|c|c|c|}
\hline \multicolumn{12}{|c|}{ bulk water } \\
\hline$\omega_{1}\left(\mathrm{~cm}^{-}\right.$ & $\mathrm{I}_{1}$ & $\Gamma_{1}$ & $\omega_{2}$ & $\mathrm{I}_{2}$ & $\Gamma_{2}$ & $\omega_{3}$ & $\mathrm{I}_{3}$ & $\Gamma_{3}$ & $\omega_{4}$ & $\mathrm{I}_{4}$ & $\Gamma_{4}$ \\
\hline 3080 & 16.7 & 179.2 & 3248 & 241.2 & 283.2 & 3409 & 64.9 & 167.8 & 3544 & 49.1 & 175.2 \\
\hline \multicolumn{12}{|c|}{ bulk $\mathrm{BaCl}_{2} 0.01 \mathrm{M}$} \\
\hline 3080 & 16.7 & 182.3 & 3248 & 241.3 & 283.2 & 3409 & 65.0 & 167.8 & 3544 & 49.1 & 175.2 \\
\hline \multicolumn{12}{|c|}{ bulk $\mathrm{BaCl}_{2} 0.2 \mathrm{M}$} \\
\hline 3079 & 23.2 & 225.1 & 3235 & 205.1 & 259.8 & 3405 & 98.6 & 187.1 & 3550 & 44.1 & 170.0 \\
\hline \multicolumn{12}{|c|}{ bulk $\mathrm{BaCl}_{2} 1 \mathrm{M}$} \\
\hline 3110 & 99.1 & 273.1 & 3257 & 180.2 & 255.0 & 3403 & 113.8 & 177.6 & 3547 & 49.3 & 164.7 \\
\hline \multicolumn{12}{|c|}{ bulk $\mathrm{CaCl}_{2} 0.01 \mathrm{M}$} \\
\hline 3081 & 12.1 & 162.3 & 3247 & 226.8 & 282.1 & 3408 & 58.6 & 167.5 & 3541 & 46.4 & 185.0 \\
\hline \multicolumn{12}{|c|}{ bulk $\mathrm{CaCl}_{2} \mathrm{O.2} \mathrm{M}$} \\
\hline 3083 & 23.1 & 231.2 & 3241 & 215.7 & 272.8 & 3398 & 71.7 & 174.1 & 3538 & 48.8 & 181.5 \\
\hline \multicolumn{12}{|c|}{ bulk $\mathrm{CaCl}_{2} 1 \mathrm{M}$} \\
\hline 3110 & 87.9 & 270.9 & 3257 & 171.9 & 251.7 & 3401 & 98.1 & 169.2 & 3541 & 55.9 & 174.9 \\
\hline \multicolumn{12}{|c|}{ bulk $\mathrm{MgCl}_{2} 0.01 \mathrm{M}$} \\
\hline 3085 & 5.8 & 160.0 & 3245 & 253.1 & 290.2 & 3409.3 & 63.6 & 171.5 & 3547 & 46.2 & 180.6 \\
\hline \multicolumn{12}{|c|}{ bulk $\mathrm{MgCl}_{2} 0.2 \mathrm{M}$} \\
\hline 3082 & 23.0 & 230.2 & 3241 & 215.7 & 273.0 & 3398 & 71.7 & 174.0 & 3538 & 48.8 & 181.5 \\
\hline \multicolumn{12}{|c|}{ bulk $\mathrm{MgCl}_{2} 1 \mathrm{M}$} \\
\hline
\end{tabular}




\begin{tabular}{|c|c|c|c|c|c|c|c|c|c|c|c|}
\hline \multicolumn{12}{|c|}{ MCM-41 water } \\
\hline$\omega_{1}\left(\mathrm{~cm}^{-}\right.$ & 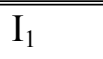 & 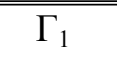 & 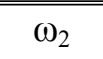 & $\mathrm{I}_{2}$ & $\Gamma_{2}$ & $\omega_{3}$ & $\overline{I_{3}}$ & 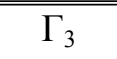 & $\omega_{4}$ & 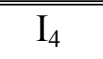 & 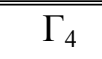 \\
\hline 3093 & 4.5 & 163.2 & 3235 & 42.7 & 220 & 3396 & 30.4 & 184.3 & 3540 & 19.1 & 196.6 \\
\hline \multicolumn{12}{|c|}{ MCM-41 $\mathrm{BaCl}_{2} 0.01 \mathrm{M}$} \\
\hline 3096 & 6.5 & 167.3 & 3238 & 31.1 & 220 & 3398 & 18.6 & 186.6 & 3545 & 11.9 & 195.2 \\
\hline \multicolumn{12}{|c|}{$\mathrm{MCM}-41 \mathrm{BaCl}_{2} 0.2 \mathrm{M}$} \\
\hline 3105 & 15 & 176.3 & 3231 & 23.5 & 188.9 & 3383 & 40.2 & 198.8 & 3535 & 20.7 & 205.4 \\
\hline \multicolumn{12}{|c|}{ MCM-41 $\mathrm{BaCl}_{2} 1 \mathrm{M}$} \\
\hline 3095 & 25.1 & 290 & 3230 & 18.4 & 189.4 & 3390 & 39.0 & 214 & 3555 & 12.6 & 187.0 \\
\hline \multicolumn{12}{|c|}{$\mathrm{MCM}-41 \mathrm{CaCl}_{2} 0.01 \mathrm{M}$} \\
\hline 3093 & 8.3 & 160 & 3240 & 27.2 & 212.6 & 3400 & 20.3 & 181.3 & 3545 & 12.2 & 204.1 \\
\hline \multicolumn{12}{|c|}{$\mathrm{MCM}-41 \mathrm{CaCl}_{2} 0.2 \mathrm{M}$} \\
\hline 3098 & 8 & 164.8 & 3235 & 20.3 & 197.1 & 3395 & 20.6 & 189.4 & 3545 & 11.5 & 196.2 \\
\hline \multicolumn{12}{|c|}{ 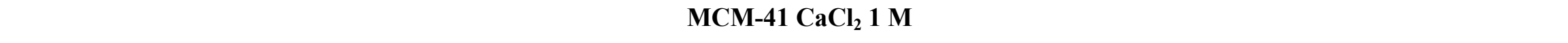 } \\
\hline 3080 & 9.1 & 217.6 & 3225 & 17.7 & 195.5 & 339, & 27 & 210 & 3500 & 10.9 & 188.9 \\
\hline \multicolumn{12}{|c|}{$\mathrm{MCM}-41 \mathrm{MgCl}_{2} 0.01 \mathrm{M}$} \\
\hline 3090 & 9.0 & 155 & 3240 & 26.4 & 205.6 & 3400 & 20.7 & 181.2 & 3545 & 13.9 & 202.4 \\
\hline \multicolumn{12}{|c|}{ MCM-41 $\mathrm{MgCl}_{2} 0.2 \mathrm{M}$} \\
\hline 3105 & 10.2 & 154.9 & 3228 & 28.9 & 177.0 & 3385 & 32.2 & 189.0 & 3540 & 21.3 & 184.9 \\
\hline \multicolumn{12}{|c|}{$\mathrm{MCM}-41 \mathrm{MgCl}_{2} 1 \mathrm{M}$} \\
\hline 3105 & 23.9 & 258.7 & 3232 & 29 & 193.2 & 3390 & 48.8 & 190.3 & 3545 & 13.9 & 189.4 \\
\hline
\end{tabular}




\begin{tabular}{|c|c|c|c|c|c|c|c|c|c|c|c|}
\hline \multicolumn{12}{|c|}{ MCM-41-1 water } \\
\hline$\omega_{1}\left(\mathrm{~cm}^{-}\right.$ & $\mathrm{I}_{1}$ & $\Gamma_{1}$ & $\omega_{2}$ & $\mathrm{I}_{2}$ & $\Gamma_{2}$ & $\omega_{3}$ & $\mathrm{I}_{3}$ & $\Gamma_{3}$ & $\omega_{4}$ & $\mathrm{I}_{4}$ & $\Gamma_{4}$ \\
\hline 3080 & 7.8 & 152.6 & 3225 & 37.9 & 196.9 & 3388 & 28.9 & 191.7 & 3541 & 15.7 & 199.5 \\
\hline \multicolumn{12}{|c|}{ MCM-41-1 $\mathrm{BaCl}_{2} \mathbf{0 . 0 1} \mathrm{M}$} \\
\hline 3088 & 11.0 & 174.1 & 3245 & 27.7 & 211.2 & 3400 & 28.8 & 172.9 & 3540 & 18.7 & 220.1 \\
\hline \multicolumn{12}{|c|}{ MCM-41-1 $\mathrm{BaCl}_{2} 0.2 \mathrm{M}$} \\
\hline 3098 & 11.5 & 154.4 & 3222 & 19.7 & 168.1 & 3378 & 35.8 & 215.7 & 3550 & 14.1 & 197.1 \\
\hline \multicolumn{12}{|c|}{ MCM-41-1 $\mathrm{BaCl}_{2} 1 \mathrm{M}$} \\
\hline 3108 & 31.5 & 301.1 & 3237 & 22 & 199.8 & 3382 & 53.2 & 223 & 3548 & 14.0 & 198.0 \\
\hline \multicolumn{12}{|c|}{ MCM-41-1 $\mathrm{CaCl}_{2} 0.01 \mathrm{M}$} \\
\hline 3098 & 8.5 & 182.2 & 3242 & 33.8 & 233.9 & & 21.7 & 184.1 & 3548 & 14.0 & 194.7 \\
\hline \multicolumn{12}{|c|}{$\mathrm{MCM}-41-1 \mathrm{CaCl}_{2} 0.2 \mathrm{M}$} \\
\hline 3105 & 14.2 & 162.6 & 3230 & 27.8 & 175.0 & 3385 & 43.0 & 196.2 & 3545 & 18.2 & 186.4 \\
\hline \multicolumn{12}{|c|}{ MCM-41-1 CaCl $21 \mathrm{M}$} \\
\hline 3108 & 30.3 & 241.5 & 3236 & 24.8 & 163.7 & 3380 & 53.1 & 153.8 & 3540 & 22 & 187.5 \\
\hline \multicolumn{12}{|c|}{ MCM-41-1 $\mathrm{MgCl}_{2} 0.01 \mathrm{M}$} \\
\hline 3098 & 7.0 & 239.4 & 3397 & 28.1 & 270.5 & 3397 & 18 & 200 & 3545 & 12.3 & 203.4 \\
\hline \multicolumn{12}{|c|}{ MCM-41-1 $\mathrm{MgCl}_{2} 0.2 \mathrm{M}$} \\
\hline 3101 & 9.9 & 156.1 & 3230 & 20.1 & 174.7 & 3383 & 28.3 & 195.3 & 3540 & 15.1 & 196.2 \\
\hline \multicolumn{12}{|c|}{ MCM-41-1 $\mathrm{MgCl}_{2} 1 \mathrm{M}$} \\
\hline 3105 & 27.1 & 260.7 & 3233 & 32 & 188.4 & 3383 & 49.8 & 197.8 & 3545 & 15 & 173.5 \\
\hline
\end{tabular}


